\title{
A Study on Double Gap Magnetic Head Positioning System for Hard Disk Drive
}

\author{
Satoru SEKO, Toru TAKEDA
}

Sony Corp. , 6-7-35 Kitashinagawa, Shinagawa-ku, Tokyo 141 Japan

\begin{abstract}
A compensation method of write off track using piggy-backed 2gap head on hard disk drive isdiscussed. The read and write gap offset of piggy-backed $2 \mathrm{gap}$ head is formulated, and the measurement method for 2 gap HDA is introduced. The residual tracking error after offset-compensation is analyzed. Finally, a linear interpolation compensation is proposed, and the theoretical residual error is estimated as less than $0.1 \mu \mathrm{m}$.
\end{abstract}

\section{INTRODUCTION}

One of the dfficulties in using MR head is R/W track center offset caused by the skew angle of the head on swing arm. Conventionally, a narrow track width on read gap on a wider written track widh was used to resolve the gap center miss alignment between the two gaps.

K.Chopra[1] showed that these alignment errors limit the allowable track densities, and that the compensation by active repositioning of the transducer between read and write operations would be necessary in higher track densities. However it seems that little attention has been given to the residual error assuming such active repositioning process.

In this paper, residual positioning errors are formulated, and a new micro-jog compensation method is proposed to achieve higher track densities more than 200 TPMM (5000 TPI) introducing both precise offset-prediction and offset servo. It is also shown that the residual positioning error could be less than $0.1 \mu \mathrm{m}$ introducing this compensation method.



Fig.1 A Sketch of Piggy-backed MR/Ind Head in Hard Disk Drive

\section{GEOMETRICAL ANALYSIS OF R/W GAP OFFSET}

\section{A. $R / W$ Head Center Offset Model}

R/W Head Center Offset is caused by two factors, miss alignment $\delta$ in production process and skew angle between track direction and arm direction.

The miss alignment $\delta$ is caused by the miss alignment of the two masks, one is for read element and another is for write element.

Figure 2 is a definition for the miss alignment $\delta$ between Read gap track center and Write gap track center.

The skew angle between track direction and arm drection depends on the position of swing arm as shown in Fig.3. Due to this skew angle and the distance between write gap and read gap, a piggy-backed write gap on a swing arm runs on slightly larger radius tracks than read gap runs. The difference in radius causes a $R / W$ track center offset.

Figure 4 shows R/W head center offset when the gaps follow a track with skew angle $\theta_{\mathrm{S}}$.

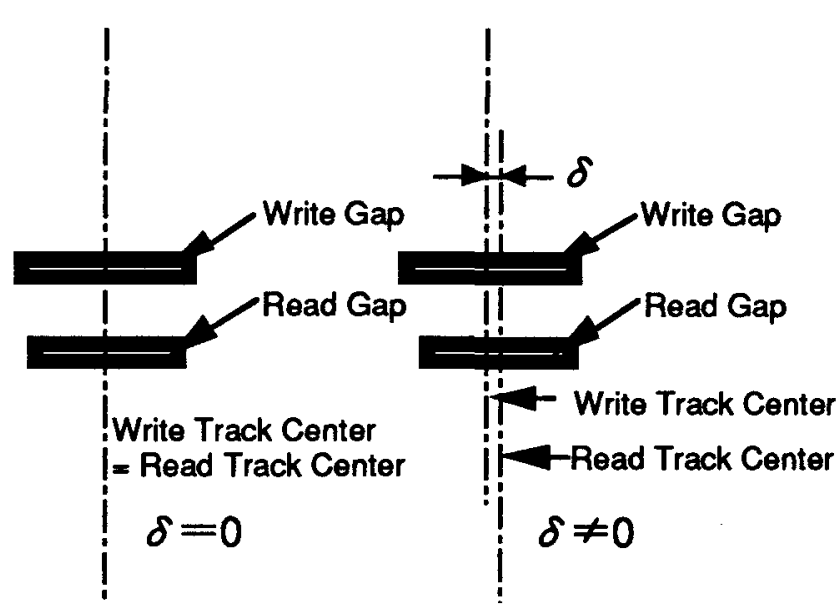

Fig.2 Read gap and Write gap with miss alignment $\delta$ in production process 




Fig.3 Skew Angle " $\theta \mathrm{s}$ " caused by Swing Arm

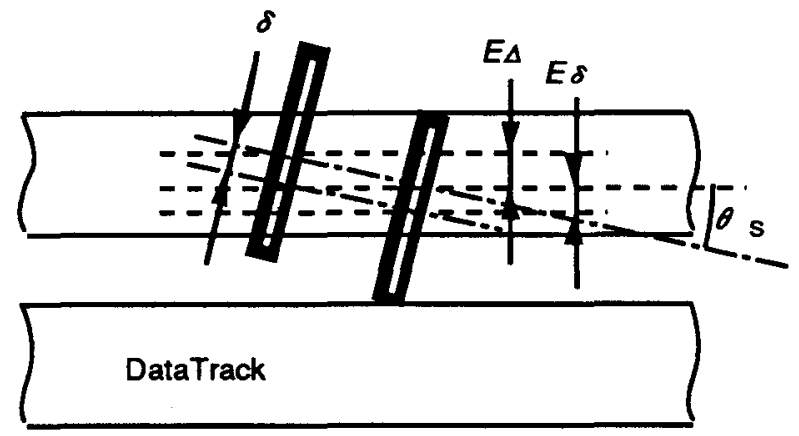

Fig.4 R/W Track Center Offset caused by Skew Angle

The total R/W track center offset value $E(t)$ is to be expressed as follows.

$$
\begin{aligned}
E(r) & =E_{\delta}(r)+E_{\Delta}(r) \\
& =\delta \cos \left[\theta_{\mathrm{B}}+\sin ^{-1}\left(\frac{r^{2}+l_{2}^{2}-l_{1}^{2}}{2 r l_{2}}\right)\right]+\Delta \sin \left[\theta_{\mathrm{B}}+\sin ^{-1}\left(\frac{r^{2}+l_{2}^{2}-l_{1}^{2}}{2 r l_{2}}\right)\right]_{(1)}
\end{aligned}
$$

where $1_{1}$ : Distance between Motor Spindle \& Arm Pivot,
$I_{2}:$ Arm Length,

$r$ : Radius of the Track,

$\theta_{S}:$ Head Skew Angle,

$\theta_{B}:$ Head Bent Angle,

$\delta$ : Miss alignment caused by R/W alignment error in mask process,

$\Delta:$ Layers thickness between Write gap and Read gap.

Based on the formula above, we calculated the R/W head center offsets, $E_{\Delta}(r)$ and $E_{\delta}(r)$, on position radius r, assuming 2.5 " form factor,

such as

$I_{\mathrm{ID}}=16(\mathrm{~mm}), \quad T_{\mathrm{OD}}=30(\mathrm{~mm}), \quad \theta_{\mathrm{B}}=0,1_{1}=40(\mathrm{~mm})$, and $l_{2}=36,40,44(\mathrm{~mm})$.

Figure 5 shows $E_{\Delta}(t)$ vs. radial position assuming $\Delta$ $=4.5 \pm 0.5(\mu \mathrm{m})$. It tells that the $\mathrm{R} / \mathrm{W}$ offset value caused by skew angle can be 1 to $2 \mu \mathrm{m}$ at maximum.

Figure 6 shows $E_{\delta}(i)$ vs. radial position assuming $\delta$ $=0.5(\mu \mathrm{m})$. It shows that $\mathrm{R} / \mathrm{W}$ head center offset $E_{\delta}(t)$ almost equals to miss alignment value $\delta$.



Fig.5 Theoretical curve of R/W Track center offset caused by the distance " $\Delta$ " between Read gap and Write gap. 


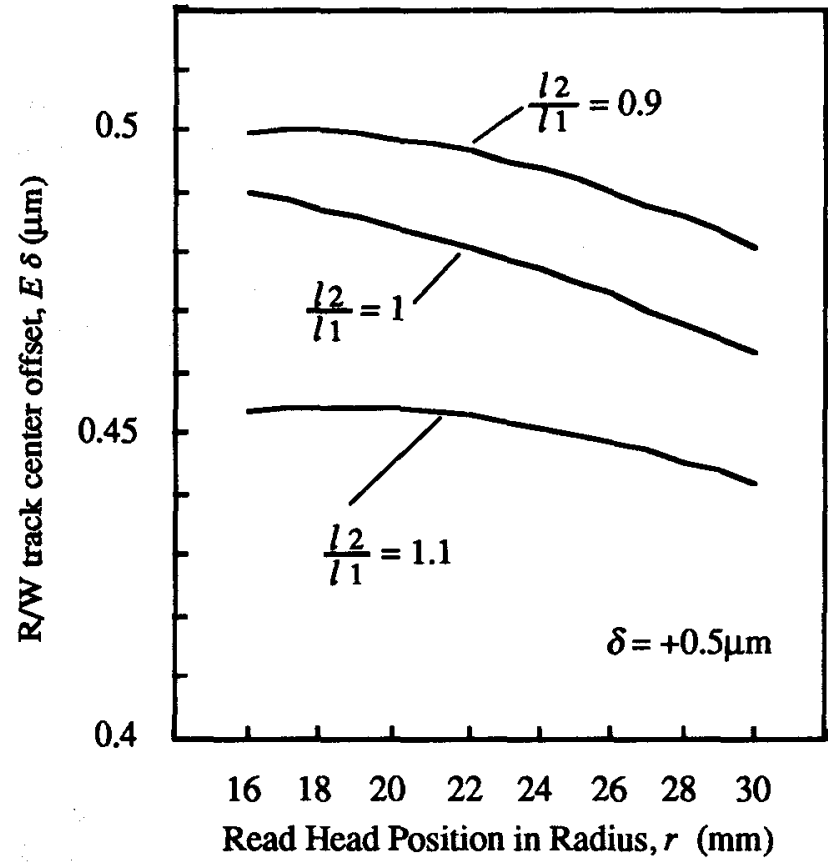

Fig.6 Influence of miss alignment between Read gap center and Write gap center on $R / W$ track center offset

Figure 5 and Fig.6 show that such large R/W gap center offset as $2 \mu \mathrm{m}$ maximum in OD caused by both $\Delta$ and $\delta$ can not be negligible on namower track pitch around $5 \mu \mathrm{m}$.

Consequently the micro jog compensation on higher track densities more than 200 TPMM (5000 TPI) will be essential.

\section{B. Miss Alignment Compensation by Offset Servo}

$\mathrm{R} / \mathrm{W}$ center offset should be canceled by micro-jog compensation according to the theoretically predicted offset value. However, a certain error necessarily remains due to the following reasons.

- difference between design value and actual value on $\Delta$ or $\delta$.

- measurement errors mingled in $\mathrm{R} / \mathrm{W}$ offset measuring process.

The error caused by the first origin would be more than $0.5 \mu \mathrm{m}$ if the dispersion of $\Delta$ or $\delta$ were neglected.

We take no notice of the second origin in this paper because the errors can be reduced by well-onsidered measuring methods.

It is concluded that the individual measurements to obtain the values for compensations are unavoidable for such precision tracking as within $0.5 \mu \mathrm{m}$.

\section{OFFSET SER VOCOMPENSATION USING LINEAR INTERPOLATION}

A simple offset compensation method is proposed to perform precise compensation in every radius on every drive. It is realized easily by a Digital Signal Processor (DSP), using linear interpolation.

\section{A. Implementation of Offset Servo}

The compensation procedure is as follows.

step1: Measure the effective track center offset values between write gap and read gap at ID and at OD respectively during power on initialization on each surface.

step2: Obtain current track radius from current track number using some algorithm.

step3: Using the DSP, interpolate track center offset value $E(r)$ in the current track radius $r$, based on the two points $E\left(r_{\mathrm{ID}}\right)$ and $E\left(I_{\mathrm{OD}}\right)($ track center offset value measured at ID or OD ) linearly.

The calculation can be simplified in this method as there is no need to use (4).

As this calculation and operation can be done existing servo DSP, no significant cost-up will be necessary.

There are various ways to measure the $R / W$ center offset at a certain radius. To show that the measurement of track center offset is possible, we note an example of the $R / W$ offset measuring methods as follows.

\section{AR/W offset measuring method}

[1] Make read gap follow the center of a track.

[2] Write and fill the track with data.

[3] Read the track and verify the data. Get the error rate on the head position.

[4] Give a micro jog by biasing the position reference in servo circuit, and get the error rate on new head position.

[5] In the same way, giving various micro jogs, store the error rates being dependent on the head positions.

[6] Find the position on which the center of balance of exror rate curve exists. The position value is exactly what we need.

The accuracy of the measurement can be improved if enough repetiton times are employed 


\section{B. Linear interpolation accuracy}

Theoretical residual error has been calculated as shown in Fig.7 for verifying the accuracy of this linear interpolation method. $\left(I_{1}=40 \mathrm{~mm}, \theta_{\mathrm{B}}=0, \Delta=5 \mu \mathrm{m}\right.$, $\left.-0.5 \leqq \delta \leqq+0.5(\mu \mathrm{m}), 0.9 \leqq 1_{2} / l_{1} \leqq 1.1\right)$

It shows that the residual error is less than $0.1 \mu \mathrm{m}$ in every radus and the error hardy depends on the value of $\delta$.

These results prove that the linear interpolation between the two points of ID and OD gives a good approximation as compensation values for each radial position, and that the alignment error of MR/Inductive head center does not bring serious problem into residual tracking error. Considering those results, following compensation algorithm will give us accurate compensation for micro-jog problem, without complex calculation.



Read Head Position in Radius, $r$ (mm)

Fig.7 Residual errors after Offset-compensation using the Linear Interpolation System.

\section{CONCLUSION}

A piggy-backed two gap MR/Inductive head offset was analyzed, and it was shown that simple linear interpolation calculation in servo DSP can compensate both piggy-back distance error and the gap center offset error. This kind of micro-jog compensation seems to be powerful aid to realize higher track density up to 1000 TPMM(25kTPI) in near future in the field of small form factor hard disk dives.

\section{REFERENCES}

[1] K.Chopra,G.M.Gram mens, R.E.Weinstein," Track Density Constraints in the Application of MR Head Technology, "IEEE Trans.Magn., vol.28, No.5, pp2728-2730 (1992)

[2] J.B.Baas,H.M.Melbye, "A Predictor Algorithm for Off-Track Read Capability," IEEE Trans.Magn., vol.29, No.6,pp3987-3989(1993)

\section{[APPENDIX]}

\section{Formulation of the R/W track center offset}

Skew angle $\theta_{\mathrm{S}}$ can be formulated as

$$
\theta_{\mathrm{S}}(r)=\theta_{\mathrm{B}}+\sin ^{-1}\left(\frac{r^{2}+l_{2}^{2}-l_{1}^{2}}{2 r l_{2}}\right)
$$

where $I_{1}$ : Distance between Motor Spindle \& Arm Pivot,

$1_{2}:$ Am Length

$r$ : Radius of the Track,

$\theta_{\mathrm{S}}:$ Head Skew Angle

$\theta_{\mathrm{B}}:$ Head Bent Angle.

The R/W track center offset in radius $r, E_{\delta}(r)$ and $E_{\Delta}(r)$ can be expressed as

$$
\begin{aligned}
& E_{\delta}(r)=\delta \cos \theta_{S}(r) \\
& E_{\Delta}(r)=\Delta \sin \theta_{S}(r)
\end{aligned}
$$

where $\delta$ : Miss alignment caused by R/W alignment error in mask process.

$\Delta:$ Layers thickness between Write gap and Read gap

Thus, total $\mathrm{R} / \mathrm{W}$ track center offset value " $E_{\delta}+E_{\Delta}$ " can be expressed as (1). 